\title{
针对初级汉语学习者的汉字书写偏误 Analisis Kesalahan Penulisan Aksara Mandarin Pada Pembelajar Bahasa Mandarin Pemula
}

\author{
Silvia \\ Program Studi Bahasa Mandarin S1, Sekolah Tinggi Bahasa Harapan Bersama \\ E-mail: fangg77@qq.com
}

\begin{abstract}
摘要
听说读写是汉语教学中的四大技能, “写”之所以排在第四, 就代表了在四个技能 中, 把握好 “写” 是最难的。考虑以上因素, 文章从学生的书写情况出发, 对学生的作 业和练习进行分析和统计, 发现学生在笔画, 笔顺, 和笔向偏误最高, 其次在部件及部 首及字形和字音等方面, 最后, 为了减少偏误, 笔者认为学生首先必须重视汉字的教学, 对传统的笔画笔顺有扎实的认识，同时，学生也需要反复练习和操练，才能减少和避免 汉字的偏误。
\end{abstract}

关键词：汉字书写；偏误分析；笔画笔顺；结构。

\begin{abstract}
Abstrak
Mendengar, berbicara, membaca dan menulis adalah empat keterampilan utama dalam pengajaran bahasa Mandarin. Alasan "menulis" menempati urutan keempat, dikarenakan di antara keempat keterampilan tersebut, menguasai "menulis" adalah yang paling sulit. Mempertimbangkan faktor-faktor di atas, karya tulis ini dimulai dari kondisi menulis siswa, menganalisis tugas dan latihan, serta menemukan kesalahan terbanyak dalam urutan guratan dan arah goresan, kemudian komponen, radikal dan pengucapan. Untuk mengurangi kesalahan tersebut, penulis mempercayai bahwa pembelajar terlebih dahulu harus memperhatikan pengajaran aksara Mandarin dan memiliki pemahaman yang kuat tentang urutan guratan tradisional. Pada saat yang sama, siswa juga perlu berlatih berulang kali untuk mengurangi dan menghindari kesalahan dalam penulisan aksara Mandarin.
\end{abstract}

Kata kunci: Penulisan aksara Mandarin; Analisis kesalahan; Urutan guratan; Struktur 


\section{一 前言}

根据人们常使用的《新华字典》，中国的汉字大约有 10.000 多个字，其中常用的汉 字就有 1000 多个。在书写方面, 中国人自己在日常的书写过程中有时还会出现一些偏 误, 而对于零基础的汉语学习者而言, 要学会正确地书写汉字, 从以往到现在都是一个 大难题，导致这些偏误的原因也非常多样。

首先, 从汉字本身来说, 汉字与其他文字截然不同, 汉字有独特的笔画笔顺和结构, 而且有些汉字它的字形几乎是相同的, 这些其实成为了学生为何难以掌握好汉字的原因;

其次, 从学生自身原因来说, 有些学生对汉字的字形和结构过于陌生, 有些学生对 于书写汉字的态度和习惯非常不好，从而一直重复同样的错误;

最后, 从教师自身原因来说, 教师不论在教学方法方面还是在给学生操练的时候, 多多少少会不重视和忽略汉字的基本知识, 缺乏反复练习等等, 导致学生可能在学习过 程中出现了许多偏误。

\section{二 研究对象及方法}

本文的研究对象确定为 23 名西加华文教育协调机构（Badan Koordinasi Pendidikan Bahasa Mandarin Kalimantan Barat）的学生，这些学生虽说没考过 HSK，但已经学了六 个多月汉语了。本文主要通过收集学生在第一学期的汉字课作业以及练习来进行详细分 析, 决定选取第一学期作为研究资料的原因是因为想知道在最初学习的时候, 学生和教 师存在着哪些问题。

本文使用的是定量性研究方法, 确定偏误的方法是若在 29 位学生的研究材料中, 多 数出现相同的问题; 少数出现相同的问题, 但与其他错误有很大的关联, 都将视为书写 偏误。

\section{三 偏误类型分析}

笔者收集了材料之后, 分别分析出了学生书写上的几类偏误, 详细分析可阅读以下总 表。 
表 3.1 汉字书写偏误表

\begin{tabular}{|c|c|c|c|c|c|c|}
\hline 序 & $\begin{array}{c}\text { 偏误类 } \\
\text { 型 }\end{array}$ & 具体分析 & 偏误材料 & $\begin{array}{c}\text { 偏误 } \\
\text { 出现次数 }\end{array}$ & $\begin{array}{l}\text { 偏误占比 } \\
\text { (百分比) }\end{array}$ & $\begin{array}{c}\text { 总 } \\
\text { (百分比) }\end{array}$ \\
\hline 1 & $\begin{array}{l}\text { 笔顺和 } \\
\text { 笔画 }\end{array}$ & 笔顺写错 & 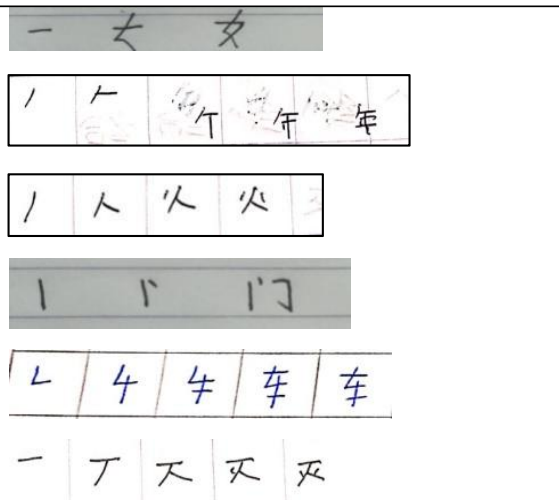 & 45 & $32.8 \%$ & \\
\hline & & 笔画写错 & 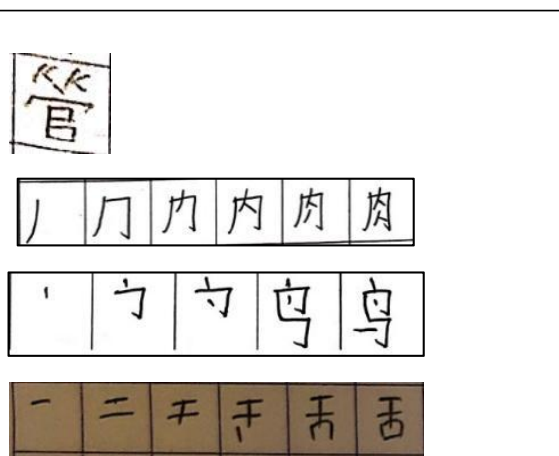 & 52 & $37.95 \%$ & \\
\hline & & $\begin{array}{l}\text { 笔画增多 } \\
\text { 或少 }\end{array}$ & 多. & 40 & $29.19 \%$ & \\
\hline & & & & 137 & $100 \%$ & $49.2 \%$ \\
\hline 2 & $\begin{array}{l}\text { 部件和 } \\
\text { 部首 }\end{array}$ & $\begin{array}{l}\text { 部件和部 } \\
\text { 首更换 }\end{array}$ & 但原因 & 45 & $66.17 \%$ & \\
\hline & & $\begin{array}{l}\text { 部件位置 } \\
\text { 更换 }\end{array}$ & $\frac{p}{\frac{P}{1}}$ & 23 & $33.82 \%$ & \\
\hline & & & & 68 & $100 \%$ & $24.4 \%$ \\
\hline
\end{tabular}




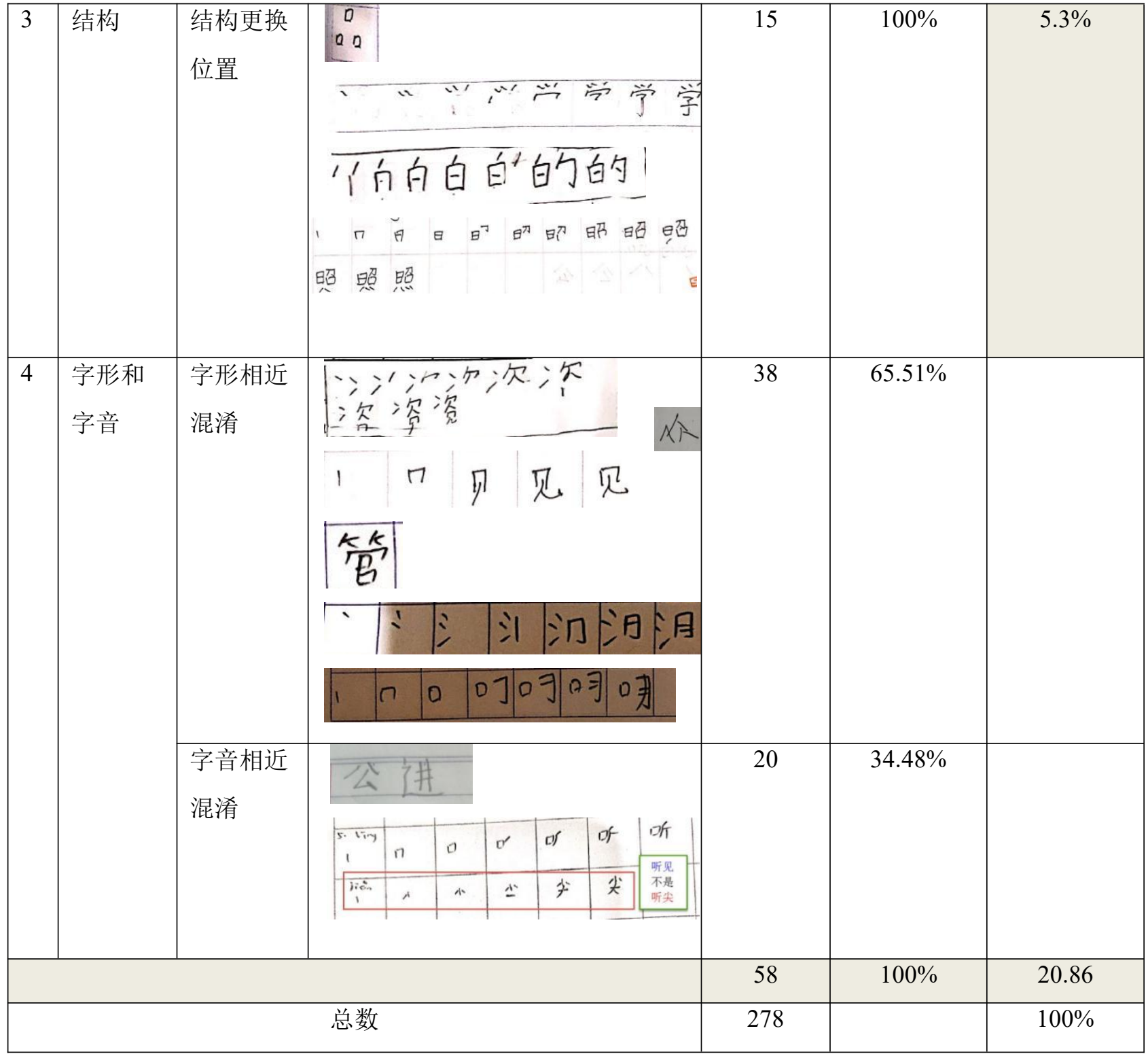

本文把书写偏误具体分析分为大致分为 4 钟，第一、笔顺和笔画偏误; 第二、部件 和部首偏误；第三、结构偏误；最后一个则是字形和字音的偏误，具体分析如下：

(1) 笔顺和笔画偏误

汉字表面看起来很复杂，但如果我们把复杂的汉字拆开，就能发现一些基本笔画， 这些汉字的笔画其实就是汉字的最小零件。这些基本笔画看似简单但如果写的过程当中 没有认真和注意的话，出现各种偏误已成为初级学习者的普遍现象。

其中的偏误就是笔顺和笔画偏误, 汉字的笔顺是有先后顺序和规律的, 从表 1 能看 出, 笔顺的错误在笔画和笔顺错误的占比高达 $32.8 \%$, 错误率 挺高的, 许多对汉语不 
熟悉的人在书写汉字的时候，最常把 “写” 当作 “画”，若要写对和写好汉字，学生 必须先掌握好规则，如：先横后坚、从上到下、先外后内、先写中间再后两边等，这些 都是通常的顺序，但汉字的的规则是不完全统一，比如：“门” 的第一个笔画是先从 “点” 开始的, 再是 “女” 字则是先从横开始, 这些不寻常的规律就是导致学生出现偏 误的原因。

另外，从汉字笔画来分析，汉字的基本笔画虽说只有 “横、坚、撇、捺、点、提、 折” , 但在写的时候, 从长短、方向和写字距离来看, 有不少汉字会有一些变化, 变形, 甚至变成了其他的笔画。有 $37.95 \%$ 的学生有笔画方面的偏误, 印尼学生最常写错的部 首就是 “竹” 如：例子中的 “管” 字，第二个和第五课笔画应该是 “横”，但学生总把 它写成印尼语里面的 “ $\mathrm{k}$ ”, 这是学生受了母语的印象导致的笔画偏误, 一些学生也总 喜欢把 “舌”字的第一个笔画写成 “舌”，再是 “乌”字，第一个笔画应该是 “潄” 而不是 “点”，有一些汉字课本的字体不明显，有的学生会很容易就被误导，所以汉字 字体应该设置为楷体。

还有另一种偏误是像上面的 “鱼” 字，在 “田” 字部分少了一笔，有时候因为对汉 字的认识不足, 再加上很多学生的学习方式是被动的, 需要等教师来布置任务, 所以学 习和练习的机会就更少了, 避免这种偏误需要学生自己更重视汉字学习, 教师要让他们 意识到学习汉字的好处及乐趣。

（2）部件和部首偏误更换

汉字的部件是笔画构成的，大部分让学生混淆的部首独体字，而是合体字，有些部 件本身就是汉字，比如：“意” 字则是 “立、日、心”三个汉字组成的，但有些部件则 是 “不成字部件”，比如：“部” 字是由 “立、口、队”三个部件组成的。刚开始接触 汉字的学生, 没有具备良好的汉字知识, 自然而然不知道 “部件” 是什么, 也不熟悉, 所以学起来非常吃力, 对汉字的记忆特别弱, 从这一点来说, 有必要一开始就让学生知 道汉字部件的作用, 这样不仅利于学生记住汉字, 而且长期以来还能大大减少书写上的 偏误。

从表 1 就可以看出学生把 “前” 字的 “月” 字写成 “用”，还有 “玩” 字的 “元” 写成 “完”，这属于部件的更换。有的把 “难” 写成 “淮” ; 有的把 “管”字的 “竹” 写成 “井”, 这些属于 部首更换字的偏误。 
（3）结构偏误

汉字的结构大部分来源于合体字, 因此合体字是学生最主要的学习重点, 合体字主 要的结构形式有左右结构、上下结构和包围结构。这三个结构还分别分成左大右小、左 小右大、上下左右混合结构、两面; 三面; 四面包围的结构, 若我们看表 1 的调查结果, 结构偏误的调查结构只有 $15 \%$, 说明了犯错的可能性并不高, “众” 字是上下左右混合 结构, 但学生却把这个字写成 “众”, 说明学生对汉字结构的认识还不深, 再是 “唯” 字应该是左小右大结构, 所以 “口” 字应该写小一些, 但学生还不认识汉汉字有这类的 结构, 他们以为汉字左右的字体大小应该是一样的, 其实不全是。再是 “如” 字, 有些 学生总是会把 “女” 和 “口” 分开写, 距离的拉开非常大, 看起来已不是一个 “如” 字。 总得来说汉字的结构挺多样的, 但若学生加常练习, 汉字结构方面的偏误是完全可以避 免的。

(4) 字形相近和字音相近混淆

字形相近也被称之为 “形近字” ，它指几个字形结构相近的字，的意思就是从字自 身的形状 (笔顺笔画) 来看非常相同, 这种偏误非常容易看出, 例如在表 1 , 学生把 “己” 和 “已”字混淆; 还有 “刀” 和 “刀” ; “人” 和 “入” ; 都属于字形相近混淆 的偏误。

另外还有字音相近混淆, 字音相近也被称 “音近字” , 指读音相同但它的字形和语 义不同。比如学生把 “工作”，写成 “工做” ; “汉字” 写成 “汉子”; 还有 “幸福” 写成 “兴福” 等, 这写都是字音相同导致的偏误, 属于字音相近混淆。从字形和字音的 偏误比率来看, 学生的偏误多数出现在字形相同方面达到了 $65.5 \%$, 说明学生更容易混 淆字形相同的字, 这类型的错误还是需要学生自己随着学习的时间和推移, 对汉字的认 识多了，才可会逐渐地减少了偏误。

\section{四 教学建议及结语}

汉字学习是学生学习汉语的主要部分之一, 因此从一开始掌握好 “写” 十分重要, 有了正确的认识的基础才有了扎实的基础。因此, 本文根据以上的偏误分析结果提出了 以下几种策略: 
(1) 学生要重视汉字的书写

学多学生只对口语有很强的学习意识, 而对汉字却有很大的不自信, 他们认为汉字 是非常难以掌握的, 教师需要让学生对汉字有更强的认识, 与此同时, 我们还需要帮助 学生树立起学好汉字的自信。教师可以把汉字背后的许多鬼力介绍给学生, 告诉学生汉 字是非常有趣和美妙; 在汉字教学的过程当中，教师可以先从最容易的汉字来入手，等 到学生慢慢学会了最基本的笔画之后，我们才开始从“横折弯钩”、“坚提”、“坚折 折钩” 进行扩展, 再慢慢地介绍出汉字的结构、部件和部首。

(2) 教师需要让学生都学到汉字的知识

汉字是非常独特的，它不仅是 “音-形-义”三个因素建立而成的，而且它最大的特 点就是可以灵活组合。最系统的汉字结构分为：笔画 $\rightarrow$ 部件 $\rightarrow$ 形旁和声旁 $\rightarrow$ 整字, 所以 教师在教学当中需要突出汉字的字音、字形及字义, 我们不能单独, 或孤立地教, 这样 可能导致学生的学习效率和结果不是很理想。再是, 许多老师只是依偎地教和强调汉字 的笔顺和笔画, 而忽略了让学生学会分清楚结构、部件、和部首是非常重要的, 若学生 对能学好和分清结构和部件, 下次学生就可以更快地记住汉字; 而学会汉字的部首以后, 他们则能分清汉字是有形旁和声旁的，如：“木” 字作为偏旁，跟它关联的字有 “树、 林、杨”，它们在意义上都跟 “木” 有关系，所以如果学生掌握了汉字的部首，可以避 免在汉字的部首方面出现偏误, 总而言之, 让学生有扎实的汉字知识是非常重要的。

(3) 教师需要让学生反复操作和练习

有一句俗话说: “熟能生巧”, 掌握好任何一种语言最重的是进行大量的练习, 不 停地练习和使用才能把别人的东西变成自己的东西。书写汉字也不例外, 只有在课堂上 多增加练习数量才会利于学生认识、理解、以及掌握汉字的规律。教师在课堂上多进行 反复操练不仅能够帮助教师考察出学生的理解程度, 还能够帮助学生认识和纠正自己的 错误, 从而减少了错误的频率。学生在操作练习的时候可以采用临摹、描写、回忆默写 法等方法。

学习汉语的过程中, 我们有 “听、说、读和写” 这四个方面, 每一个部分都不可缺 少, 都必须得到同样的重视, 要做到全局兼顾。与此同时, 还希望教师和学生在教学和 学习的过程当中不要放弃汉字的学习。在实践的过程中, 这些建议还需要教师自己加倍 关心和参与, 这样才能达到有条理、有趣以及有效率的汉字课堂。 


\section{参考文献}

[1]赵金铭. 对外汉语教学概论 [M]. 北京: 商务印书馆, 2009.

[2] 周健. 汉字突破 $[\mathrm{M}]$. 北京. 北京大学出版社, 2005.

[3] 郭圣林. 汉字的笔画特点与外国学生汉字笔画偏误 $[\mathrm{J}]$. 广州: 暨南大学华文学院学报 2008 (4).

[4]殷源苑. 留学生汉字书写偏误与原因分析 [J]. 南京：智库时代 2018（25）.

[5]Metta Eka Putri, Herman: 印尼峇淡慈容学校初中部教师汉字教学方法和学生汉字学 习方法调查研究 (Analisis Metode Pengajaran Aksara Mandarin Guru Dan Metode Pembelajaran Aksara Mandarin Siswa SMP Maitreyawira Batam, Indonesia)[J]. Jurnal Cakrawala Mandarin, April 2020, Volume 4, Nomor 1, halaman: 74-93.

\section{Catatan:}

Artikel pernah dipresentasikan pada Seminar Nasional APSMI 24 Oktober 2020 